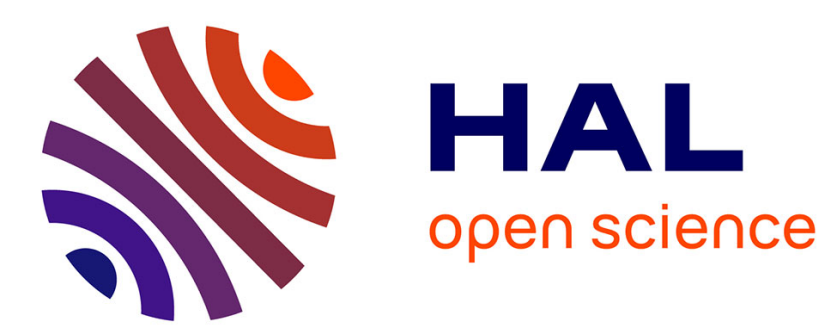

\title{
Flow of foam through a convergent channel
}

\author{
Benjamin Dollet, Claire Bocher
}

\section{To cite this version:}

Benjamin Dollet, Claire Bocher. Flow of foam through a convergent channel. European Physical Journal E: Soft matter and biological physics, 2015, 38 (11), pp.123. 10.1140/epje/i2015-15123-3 . hal-01235023

\section{HAL Id: hal-01235023 \\ https://hal.science/hal-01235023}

Submitted on 27 Nov 2015

HAL is a multi-disciplinary open access archive for the deposit and dissemination of scientific research documents, whether they are published or not. The documents may come from teaching and research institutions in France or abroad, or from public or private research centers.
L'archive ouverte pluridisciplinaire HAL, est destinée au dépôt et à la diffusion de documents scientifiques de niveau recherche, publiés ou non, émanant des établissements d'enseignement et de recherche français ou étrangers, des laboratoires publics ou privés. 


\title{
Flow of foam through a convergent channel
}

\author{
Benjamin Dollet ${ }^{1}$ and Claire Bocher ${ }^{1}$ \\ Institut de Physique de Rennes, UMR 6251 CNRS/Université Rennes 1, Campus Beaulieu, Bâtiment 11A, 35042 Rennes Cedex, \\ France
}

Received: date / Revised version: date

\begin{abstract}
We study experimentally the flow of a foam confined as a bubble monolayer between two plates through a convergent channel. We quantify the velocity, the distribution and orientation of plastic events, and the elastic stress, using image analysis. We use two different soap solutions: a sodium dodecyl sulfate (SDS) solution, with a negligible wall friction between the bubbles and the confining plates, and a mixture containing a fatty acid, giving a large wall friction. We show that for SDS solutions, the velocity profile obeys a self-similar form which results from the superposition of plastic events, and the elastic deformation is uniform. For the other solution, the velocity field differs and the elastic deformation increases towards the exit of the channel. We discuss and quantify the role of wall friction on the velocity profile, the elastic deformation, and the rate of plastic events.
\end{abstract}

PACS. XX.XX.XX No PACS code given

\section{Introduction}

Aqueous foams are metastable dispersions of gas bubbles within a continuous liquid phase, which is a solution of surfactants, which stabilise the liquid films separating adjacent bubbles from rupture [1]. Two physical parameters are crucial in the description of foams: the liquid fraction $\phi_{\ell}$, and the mean bubble area $A$ (or, equivalently, the mean bubble radius).

By opposition to bubbly liquids, foams have generally a liquid fraction low enough to be a jammed system: the bubbles are packed and share thin films. Hence, bubbles have to deform elastically by a substantial amount before flowing one past each other, and foams then acquire elastoplastic properties. They thus belong to the vast class of complex fluids, like polymers, pastes or slurries. Contrary to most other complex fluids, the microstructural item of foams, i.e. the bubble, is easy to image and its elementary micromechanics is defined unambiguously. More precisely, the elastic stress can be directly deduced from the geometry of the bubble boundaries [2], and there is a clear elementary plastic event, the so-called T1 event: it is the neighbour-swapping of four adjacent bubbles. The macroscopic fields (elastic stress, plastic deformation, and velocity) describing the mechanics of a foam as a whole then directly result from averaging the information obtained at the bubble scale. Therefore, foams (as well as dense, jammed emulsions) are model systems to understand the flow of complex fluids across the scales.

Because foams are opaque in bulk, obtaining the information at the bubble scale is difficult on three-dimensional (3D) foams and the local description of $3 \mathrm{D}$ foam flows has been performed only very recently, using fast X-ray tomography [3]. Most experimental studies have resorted to foams confined in two dimensions (2D), e.g. between two glass plates, which renders the structure of the bubbles transparent (Fig. 1b). For these confined systems, various flow geometries have been studied: parallel flows driven by the motion of side walls (Couette flows) $[4,5]$ or by a pressure gradient (Poiseuille flows) [6], or flows in more complex geometries such as past obstacles [7-9] or through constrictions $[10,11]$. Similarly, most numerical simulations of foams in pure shear [12-14], past obstacles [15-18], or through constrictions [19,20] have been performed in $2 \mathrm{D}$, with some exceptions in $3 \mathrm{D}$, limited to monodisperse foams in quasistatic regimes [21,22].

A specificity of foams confined in $2 \mathrm{D}$ by plates is the presence of friction (which we shall henceforth call "wall friction") between the moving bubbles and the fixed walls, which increases at increasing foam velocity [23]. If the velocity is low enough, the effect of wall friction remains negligible; in particular, the foam flow is quasistatic. On the other hand, at large velocity, wall friction competes with the internal stress of the foam, of elastic and viscous origin, and can even lead to the catastrophic failure of the foam, reminiscent of brittle fracture [24-26].

So far, the flow of foams or dense emulsions in a convergent channel has not been much studied, except by the groups of Earnshaw and Weeks, with a special emphasis on the local description of plastic events [27-29]. However, this configuration is particularly interesting, because the convergent flow is complex enough to include both shear and elongation, contrary to parallel flows, but simple enough to be amenable to some analytical predictions. 
Indeed, in Newtonian fluid mechanics, it is one of the very few configurations where the full Navier-Stokes equations admit an analytical solution, at least for a steady and purely radial flow $[30,31]$, with in particular an explicit expression of the velocity profile in the boundary layer at high Reynolds number $[32,33]$. This comes from the fact that for incompressible flows at velocity $\boldsymbol{v}$, the symmetry of the convergent channel (neglecting end effects) giving a radial flow: $\boldsymbol{v}=v(r, \theta) \boldsymbol{e}_{r}$ (see Fig. $1 \mathrm{~b}$ for the definition of the polar coordinates), combined with the continuity equation $\boldsymbol{\nabla} \cdot \boldsymbol{v}=0$, yields a particularly simple expression for the velocity field:

$$
v=\frac{f(\theta)}{r}
$$

irrespective of the constitutive equation of the fluid.

In this paper, our goal is two-fold. First, we document the quasistatic flow of a foam in a convergent channel, when wall friction plays a negligible role. In particular, we show that the velocity profile (1) holds, we show the clear connection between the velocity field and the distribution of plastic events, and we quantify the efficiency of plastic events as increments of deformation. Second, we study non-quasistatic flows at large wall friction, we quantify how the latter modifies both the velocity profile (1) and the elastic stress, and we discuss these effects in the context of recent models.

\section{Materials and methods}

\subsection{Setup}

We have adapted the setup described in [11]. The foam flows in a Hele-Shaw cell, made of two horizontal glass plates of length $170 \mathrm{~cm}$ and width $32 \mathrm{~cm}$, separated by a gap $h=2 \mathrm{~mm}$ thin enough that the foam is confined as a bubble monolayer (Fig. 1b). Two slanted plastic plates of thickness $2 \mathrm{~mm}$ are inserted aside the Hele-Shaw cell, so that the remaining space within the channel constitutes the convergent channel, of opening half-angle $\alpha_{0}=26^{\circ}$ (Fig. 1b). The exit of the convergent channel has a width of $2.2 \mathrm{~cm}$, much smaller than the width at its entrance $(32 \mathrm{~cm})$.

The channel is connected upstream to a vertical chamber (Fig. 1a) in which a soap solution is fed at a prescribed flow rate $Q_{\ell}$ thanks to a pump. Nitrogen is continuously blown through injectors at the bottom of this chamber. The flow rate in each injector is independently controlled with a flow-rate controller. We identify the liquid fraction $\phi_{\ell}$ as the ratio of the liquid flow rate to the total flow rate: $\phi_{\ell}=Q_{\ell} /\left(Q_{g}+Q_{\ell}\right)$, with $Q_{g}$ the gas flow rate. The resulting foam accumulates on top of the chamber, then flows through the channel. The transit time through the whole channel is less than 10 minutes in all experiments; we do not observe significant changes of bubble size during this time, hence coarsening is negligible.

We have used two soap solutions. The first is a solution of sodium dodecyl sulfate (SDS) in ultrapure water
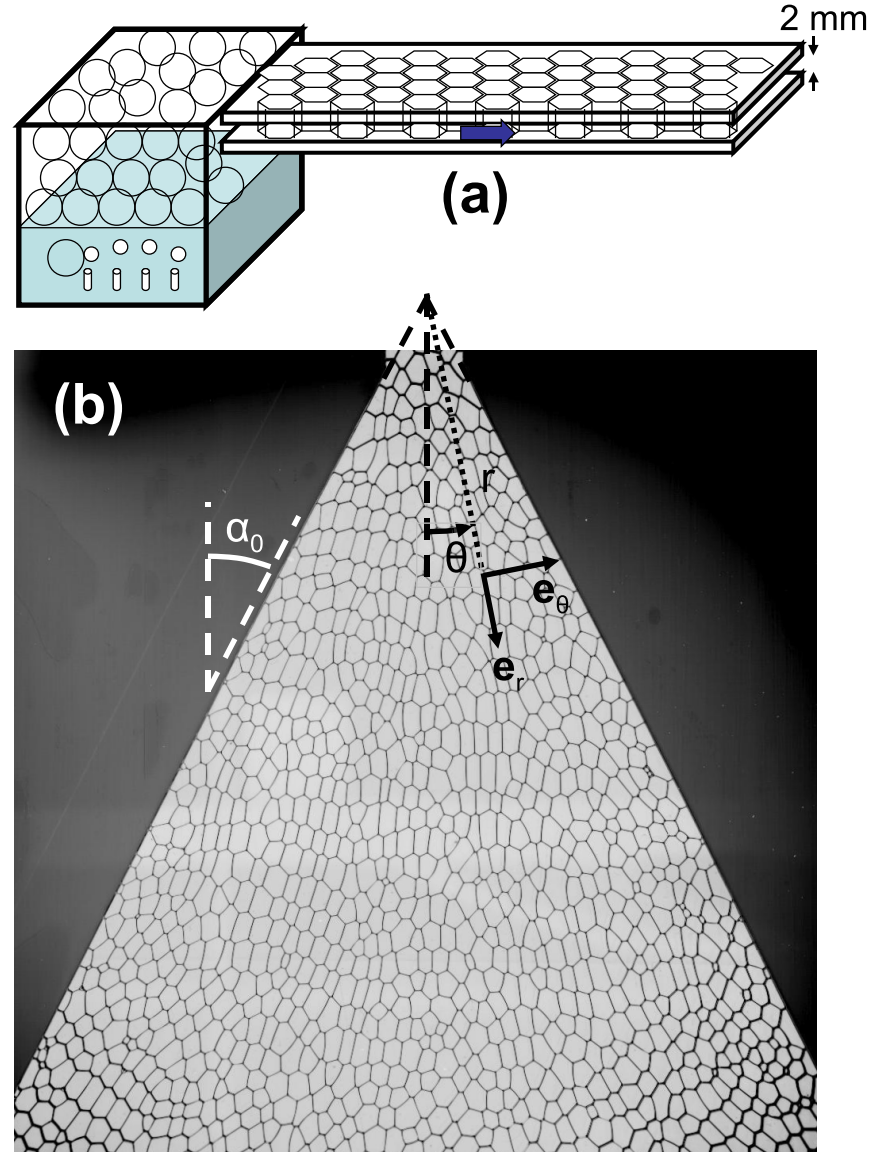

Fig. 1. (a) Sketch of the side view of the setup. For clarity, the slanted plates limiting the convergent channel have not been drawn. (b) Top view of the convergent channel, with the definition of the polar coordinates used throughout the text.

at a concentration of $10 \mathrm{~g} / \mathrm{L}$, much larger than the critical micellar concentration of SDS $(2.3 \mathrm{~g} / \mathrm{L})$ : the foam was perfectly stable and no film rupture was observed. The surface tension of this solution is $\gamma=36.8 \mathrm{mN} / \mathrm{m}$. The second solution is a mixture of sodium lauryl-dioxyethylene sulfate (SLES), cocoamidopropyl betaine (CAPB) and lauric acid (LAc), following the protocol described in [34]. Its surface tension is $\gamma=23.8 \mathrm{mN} / \mathrm{m}$. The solution of SDS has a negligible surface viscoelasticity, and the wall friction is relatively weak. On the other hand, the SLES/CAPB/LAc mixture has a large surface viscoelasticity, and the wall friction is large. We will see in Secs. 3 and 4 that this has a dramatic effect on the flow.

The contraction region is lit by a circular neon tube, giving an isotropic and nearly homogeneous illumination over most of the image. Movies of 1000 images of the foam flow are recorded with a CCD camera.

\subsection{Image analysis}

The movies are analysed using a home-made procedure fully described in [6]. Briefly, the velocity field is obtained after averaging of all the displacements of all individual 


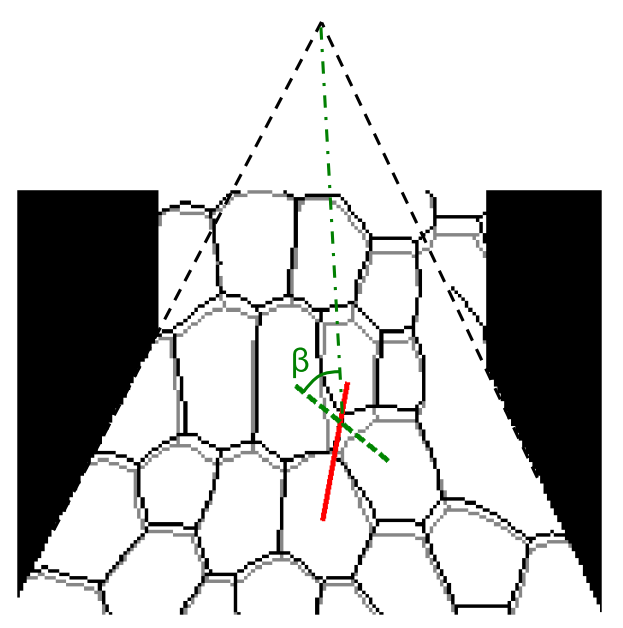

Fig. 2. Snapshot of a plastic event. Two successive skeletonised images of the foam (grey, then black) are superimposed. The link between the centres of the two bubbles which are about to lose contact is figured by the red plain line, and the link between the centres of the two bubbles that have established contact is figured by the green dashed line. The angle $\beta$ of the appearing contact with respect to the radial direction is also figured.

bubbles between consecutive frames. The plastic events, characterised by the neighbour-swapping of four adjacent bubbles (Fig. 2), are also automatically detected. This is not an easy task, especially because they are rare events, which are easily overwhelmed by artifacts if image analysis is not performed with extreme care. We estimated the relative uncertainty on the number of plastic events reported in this paper by visual inspection on a selection of images. Moreover, for dry 2D foams, the elastic stress $\sigma$ equals ([2]; see [6] for a full discussion):

$$
\sigma=2 \gamma h \rho_{\ell}\left\langle\frac{\ell \otimes \ell}{\ell}\right\rangle,
$$

where $\boldsymbol{\ell}$ designs an edge vector, i.e. a vector joining the two vertices at the extremities of a bubble edge, and $\rho_{\ell}$ is the areal density of such edges (its order of magnitude is thus $1 / A)$. The brackets stand for the average over the time interval and space zone over which the elastic stress is computed. The ability to automatically measure plastic events and elastic stress by image analysis is a major advantage of dry 2D foams for studies of local rheology.

To compute averaged quantities like the fields of velocity, elastic stress and frequency of plastic events, we use a system of polar coordinates which reflects the symmetry of the channel. The origin is the convergence point of the channel (Fig. 1b), and the angular domain covering the channel is $-\alpha_{0} \leq \theta \leq \alpha_{0}$, hence $\theta=0$ represents the axis of symmetry of the channel. The convergent channel occupies the range $r \geq 2.2 /\left(2 \sin 26^{\circ}\right)=2.5 \mathrm{~cm}$ and $r \leq 32 /\left(2 \sin 26^{\circ}\right)=36 \mathrm{~cm}$ (see Sec. 2.1 for the dimensions of the channel). We mesh the flow domain by boxes shaped as angular sectors, each with a radial length $\Delta r=7.4 \mathrm{~mm}$ and a angular opening $\Delta \theta=3.4^{\circ}$.

\section{Results}

\subsection{Reference experiment with a SDS solution}

We first report the measurements on a foam made of SDS solution, with the following control parameters: $Q_{g}=$ $100 \mathrm{~mL} / \mathrm{min}, Q_{\ell}=9.8 \mathrm{~mL} / \mathrm{min}$, hence $\phi_{\ell}=8.9 \%$, and $A=33.0 \mathrm{~mm}^{2}$. We henceforth call this experiment the "reference" experiment. We plot the velocity field in Fig. 3 and the elastic stress field in Fig. 4. As expected, the velocity field looks radial, and the velocity increases as the foam advances in the convergent channel. The elastic stress also obeys the radial symmetry: the long axes of the ellipses are aligned with the radial direction. The elastic stress does not seem to depend on the distance $r$ along the channel.

We now plot the velocity components along the symmetry axis $\theta=0$ in Fig. 5. This plot shows that the velocity component $v_{\theta}$ remains negligible compared to $-v_{r}$, and that the absolute value $\left|v_{r}\right|=-v_{r}$ of the radial velocity decreases at increasing $r$; notice that $v_{r}<0$ because the flow is convergent. Moreover, a log-log plot of $-v_{r}(r, \theta=0)$ (inset of Fig. 5) shows that its $r$-dependence is very close to a power law $-v_{r} \propto 1 / r$, which is compatible with the velocity profile (1). Concerning end effects, the graph shows a saturation of $-v_{r}(r, \theta=0)$ at the two shortest distances $r$ that we were able to measure $(r=18$ and $26 \mathrm{~mm}$ ): this is the signature of the transition from the convergent channel to the straight exit channel, which takes place at $r=25 \mathrm{~mm}$ (see Sec. 2.2). On the other hand, the entrance of the channel is at $r=36 \mathrm{~cm}$ (see Sec. 2.2), far larger than the largest distance $r$ presented on Fig. 5, hence entrance effects are probably negligible.

We have checked (data not shown) that the two main features of Fig. 5: $v_{\theta}$ negligible compared to $-v_{r}$, and $-v_{r} \propto 1 / r$, are recovered at various angular positions $\theta$ within the channel. To isolate a possible angular dependence, we therefore plot $-r v_{r}$ (averaged over 21 boxes) as a function of $\theta$, in Fig. 6. It shows that the angular velocity profile is flat, with no significant deceleration towards the side walls.

Next, we plot the elastic stress components along the symmetry axis $\theta=0$ in Fig. 7. It shows that the shear component $\sigma_{r \theta}$ is negligible compared to the diagonal components $\sigma_{r r}$ and $\sigma_{\theta \theta}$, which was expected due to symmetry. The radial component $\sigma_{r r}$ is always greater than the orthoradial one $\sigma_{\theta \theta}$, which quantifies the fact that the bubbles get elongated towards the exit of the channel (Fig. 4). More interestingly, the two diagonal components remain roughly constant across the channel. This saturation of the elastic stress means that the foam has yielded within our observation field, which suggests again that entrance effects are negligible. Indeed, entrance effects would be characterised by an elastic loading of the foam, hence we would measure an increase of $\sigma_{r r}$ and a decrease of $\sigma_{\theta \theta}$.

We now turn to plastic events. These are relatively rare events: we were able to detect $3 \times 10^{3}$ of them on the full movie, whereas there are about $10^{6}$ individual data (about $10^{3}$ bubbles on each of the 1000 images of the run) over which to average to determine velocity or elastic stress fields. Contrary to velocity and elastic stress, we 


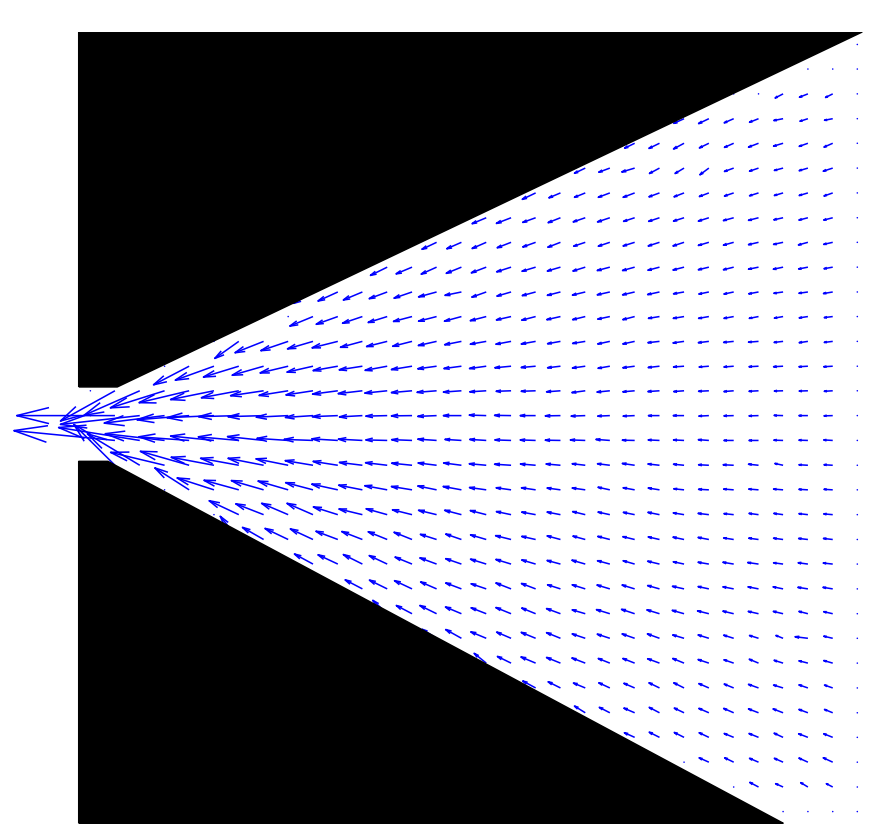

Fig. 3. Velocity field for the reference experiment.

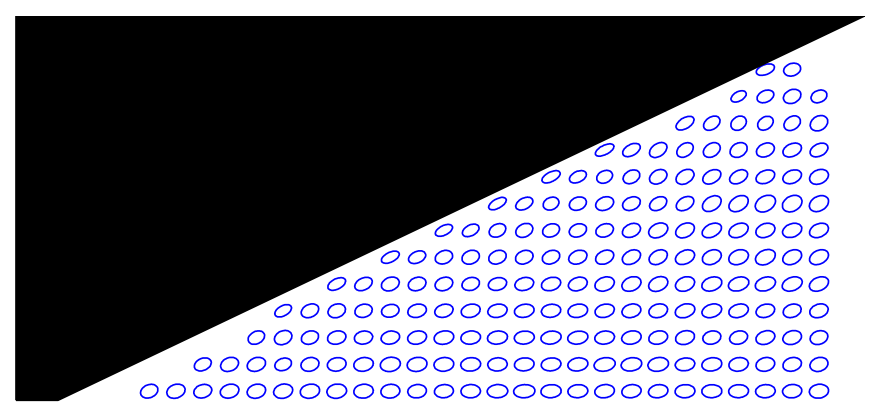
1000 000000000000000000000000000000 000000000000000000000000000000 1
1

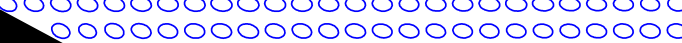
0000000000000000000000 00000000000000000000 Q 000000000000000000 10000000000000000 100000000000000 0000000000000 10000000000 000000000

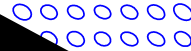
0000

Fig. 4. Elastic stress field for the reference experiment. The elastic stress (2) is a symmetric and positive tensor, hence it possesses two positive eigenvalues with orthogonal eigenvectors. It can thus represented as en ellipse, with a major (minor) axis along the direction of maximal elongation (compression)

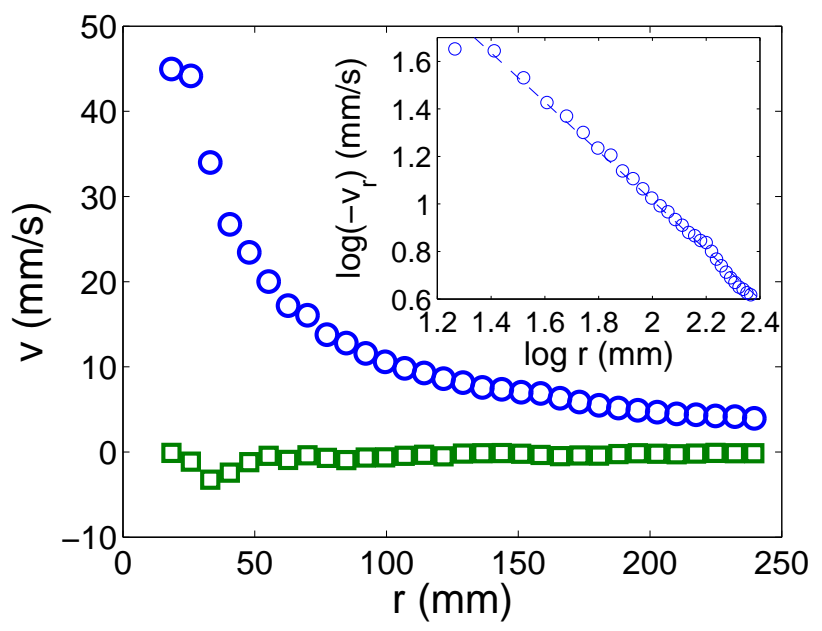

Fig. 5. Velocity components $-v_{r}$ (circles) and $v_{\theta}$ (squares) as a function of $r$, along the central axis $\theta=0$. Inset: $\log \left(-v_{r}\right)$ as a function of $\log r$, and its best linear fit, which slope is $-1.03 \pm 0.02$

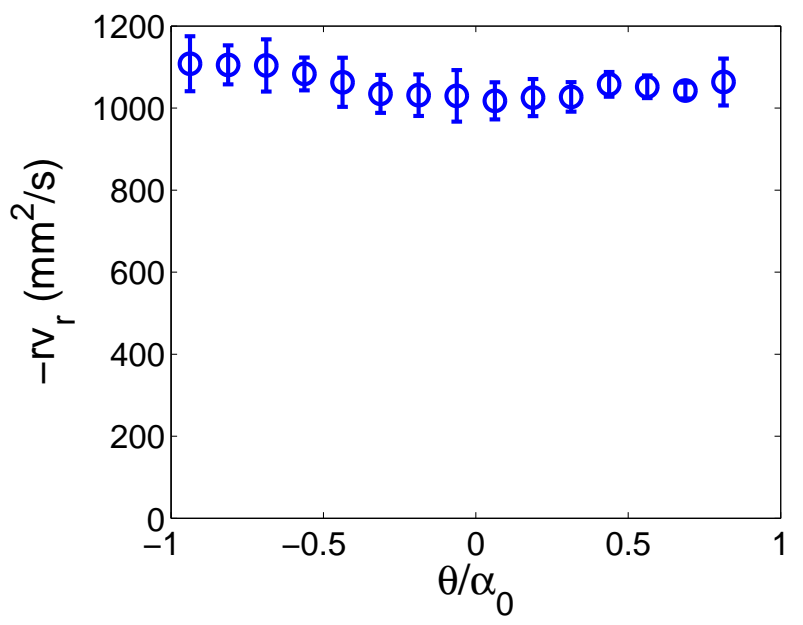

Fig. 6. Plot of $-r v_{r}$ as a function of $\theta / \alpha_{0}$. To improve the statistics, $-r v_{r}$ has been averaged over 21 boxes, for $r$ between 77 and $225 \mathrm{~mm}$. The error bars come from the standard deviation of the data between different boxes.

will thus not represent a map of plastic events. We have checked that they are equally distributed along $\theta$ (data not shown); we thus directly average over $\theta$, and define the $\mathrm{T} 1$ frequency $f_{T 1}$, in $\mathrm{mm}^{-2} \cdot \mathrm{s}^{-1}$, as the number of T1s per unit time and per unit area: $f_{T 1}(r, \theta) r \Delta r \Delta \theta$ is thus the frequency of T1s occurring in the box centred on $(r, \theta)$. We estimated a relative uncertainty of $10 \%$ on this measure. The $\mathrm{T} 1$ frequency is plotted as a function of $r$ in Fig. 8. It increases at decreasing $r$, more sharply than the velocity, until the exit of the convergent channel is reached, then strongly decreases for the shortest investigated $r$ which is in the straight exit channel. Excluding this end effect, a log-log plot of the T1 frequency shows that its $r$-dependence is compatible with a power law of exponent -2 (inset of Fig. 8). 


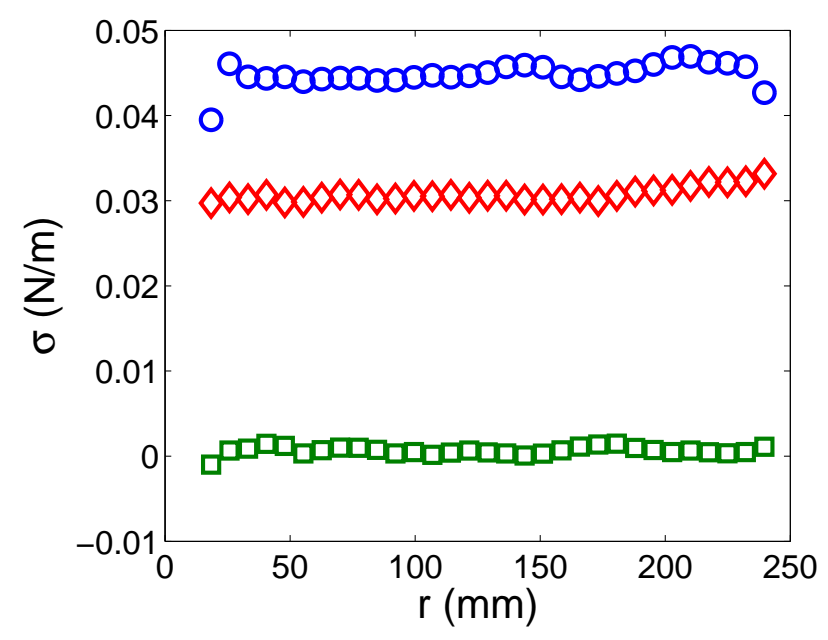

Fig. 7. Elastic stress components $\sigma_{r r}$ (circles), $\sigma_{\theta \theta}$ (lozenges) and $\sigma_{r \theta}$ (squares) as a function of $r$, along the central axis $\theta=0$.

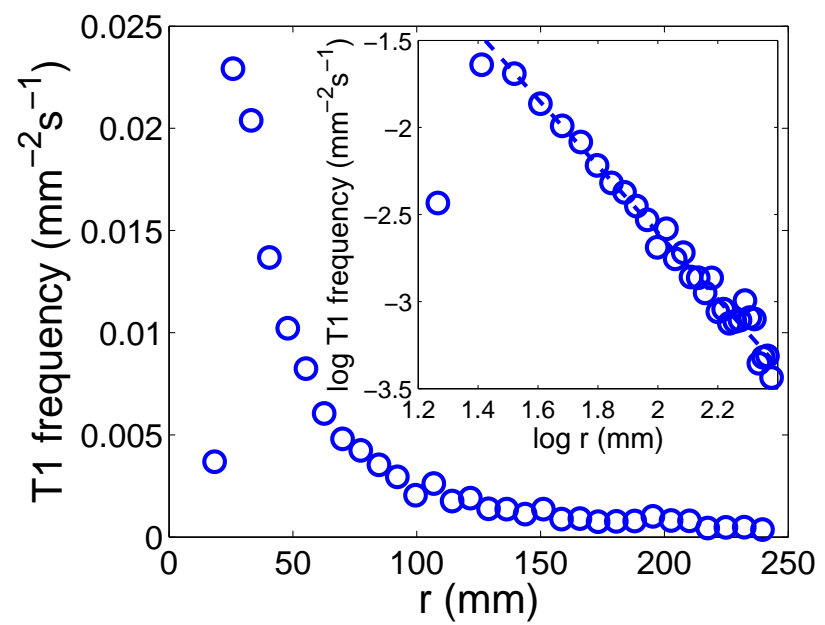

Fig. 8. T1 frequency as a function of $r$, along the central axis $\theta=0$. Inset: logarithm of the $\mathrm{T} 1$ frequency as a function of $\log r$, and its best linear fit excluding the two points at shortest $r$, which slope is $-2.10 \pm 0.14$.

Not only the spatial distribution of the plastic events matters, but also does their orientation. To quantify it, we measure the angles between the radial direction $\boldsymbol{e}_{r}$ and the direction of disappearing contacts, i.e. the direction linking two centres of neighbouring bubbles which are about to lose contact, and the direction of appearing contacts, i.e. the direction linking two centres of neighbouring bubbles which have just established contact (Fig. 2). For simplicity, we call these angles appearing and disappearing angles. Their range can be restricted to the interval $[0, \pi / 2]$. Their distribution are plotted as histograms in Fig. 9. The appearing angles have their maximum at $\pi / 2$, and the disappearing angles at 0 . Hence, the bubbles that detach tend to be aligned streamwise, and the bubbles that attach tend to be aligned spanwise. The distribution

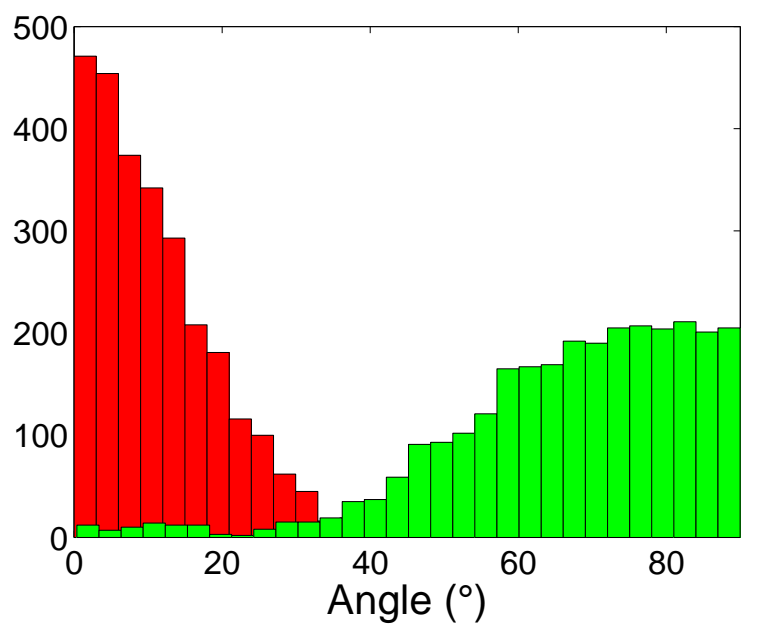

Fig. 9. Histograms of the distributions of the appearing angles (in green) and of the disappearing angles (in red).

of the appearing angles is significantly wider than the one of disappearing angles.

To conclude this presentation of the results with the SDS solution, we mention that we have performed extra experiments, changing systematically and independently the following parameters: the liquid flow rate $Q_{\ell}$ (up to $500 \mathrm{~mL} / \mathrm{min}$ ), to change the average velocity; the gas flow rate (down to $1 \mathrm{~mL} / \mathrm{min}$ ), to change the liquid fraction down to $0.5 \%$; and the bubble size, in the range $18-40$ $\mathrm{mm}^{2}$. Within this range of parameters, we have not measured any significant modification to the main features of the foam flow, namely a velocity field of the form (1), and a saturation of the elastic stress as in Fig. 7.

\subsection{Experiments with the SLES/CAPB/LAc mixture}

The results obtained with the SDS solutions suggest that wall friction remains negligible. To quantify the effects of wall friction, we now present some measurements done with the SLES/CAPB/LAc mixture. It has a large interfacial dilatational viscoelasticity [34], hence the gas/liquid interfaces are barely compressible. As a result, the bubbles tend to entrain these interfaces at their own velocity. Hence, the wetting films between the bubbles and the top, bottom and side plates are strongly sheared, and these foams develop a strong wall frictional shear stress [23].

We present an experiment with the same liquid fraction: $\phi_{\ell}=8.9 \%$ as the experiment detailed in Sec. 3.1 with the SDS solution, and a bubble area: $A=36.7 \mathrm{~mm}^{2}$ very similar (10\% larger) to that experiment. Once this foam is formed, we push it at a larger flow rate (equal to $510 \mathrm{~mL} / \mathrm{min}$ ) to enhance the effect of wall friction. This flow rate is larger than the one of the experiment presented in Sec. 3.1 (110 $\mathrm{mL} / \mathrm{min})$. However, these two experiments remain comparable: as mentioned in Sec. 3.1, we have checked that the results presented there for SDS solutions do not change significantly when the flow rate is increased to $500 \mathrm{~mL} / \mathrm{min}$. 


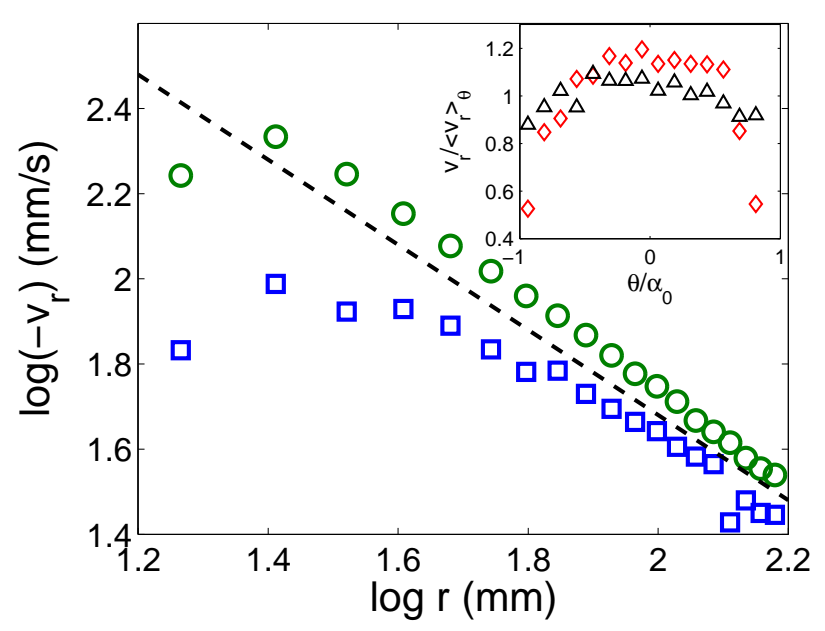

Fig. 10. Plot of $\log v_{r}$ as a function of $\log r$, for $\theta=0$ (circles) and $\theta=-25^{\circ}$ (squares). The dashed line is a guide for the eye of slope -1 . Inset: plot of $v_{r} /\left\langle v_{r}\right\rangle_{\theta}$ as a function of $\theta$, where $\left\langle v_{r}\right\rangle_{\theta}$ is the average of the data over the angle $\theta$, for $r=26 \mathrm{~mm}$ (lozenges) and $122 \mathrm{~mm}$ (triangles).

We first plot the velocity as a function of $r$, for two angular positions: the symmetry axis $\theta=0$, and an angle $\theta=-25^{\circ}$ close to the left boundary of the channel, in Fig. 10. As expected, the velocity is a decreasing function of $r$. A first difference compared with the SDS solution (Fig. 6) is that $-v_{r}$ is significantly lower close to the side walls compared to the symmetry axis. Moreover, the decrease of $-v_{r}$ as a function of $r$ is stronger at the symmetry axis. Close to the boundary, the decrease of $-v_{r}$ as a function of $r$ clearly deviates from a power law behaviour, and slows down close to the exit of the convergent channel. Moreover, the angular profile depends on $r$ : the inset of Fig. 10 shows that the larger $r$, the flatter the angular velocity profile $v_{r}(\theta)$. Therefore, the velocity field shows significant deviations from the scaling (1).

As we did for the SDS solution (Fig. 7), we plot the elastic stress components along the symmetry axis $\theta=0$ in Fig. 11. Once again, the shear component $\sigma_{r \theta}$ is negligible compared to the diagonal components $\sigma_{r r}$ and $\sigma_{\theta \theta}$, and the radial component $\sigma_{r r}$ is greater than the orthoradial one $\sigma_{\theta \theta}$. But contrary to the SDS solution, these components do not remain constant across the channel: $\sigma_{r r}$ tends to decrease, and $\sigma_{\theta \theta}$ to increase, at increasing $r$. As a consequence, the deformation of the bubbles tends to increase as they flow towards the exit of the convergent channel. The bubbles are always more deformed than with the SDS solution (Fig. 12).

Finally, we recorded the $\mathrm{T} 1$ frequency for this experiment. It turned out to be significantly noisier than for the SDS solution; the relative uncertainty was estimated to be $30 \%$, and the data for $r \geq 150 \mathrm{~mm}$ are too noisy to be exploited. We present and discuss these data in Sec. 4.2.

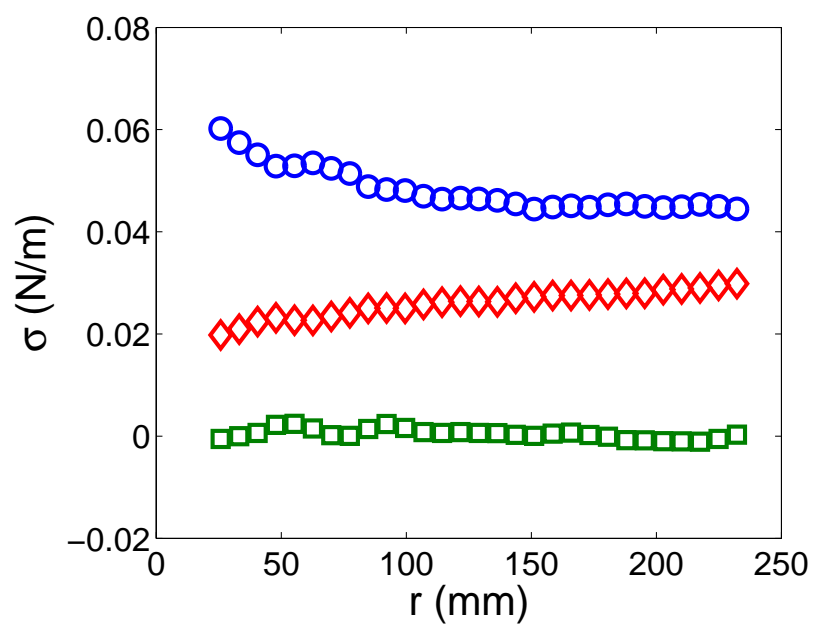

Fig. 11. Elastic stress components $\sigma_{r r}$ (circles), $\sigma_{\theta \theta}$ (lozenges) and $\sigma_{r \theta}$ (squares) as a function of $r$, along the central axis $\theta=0$. (a)

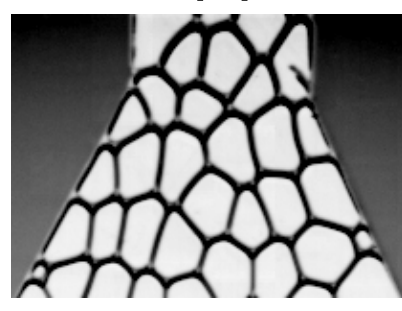

(b)

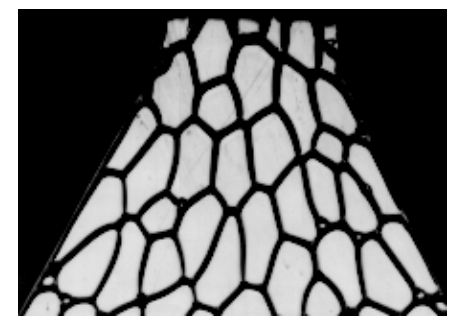

Fig. 12. Snapshots of the foam at the exit of the convergent channel, (a) for the SDS solution, (b) for the SLES/CAPB/LAc mixture. The bubbles are visibly more elongated streamwise in the latter case.

\section{Discussion}

\subsection{A model plastic flow?}

Our experimental results show that for a foam experiencing a negligible wall friction (see Sec. 4.2 for more discussion on the influence of wall friction), the kinematics of the flow in a convergent channel follows the very simple law (1) (Fig. 3 and 5).

This result does not come as a surprise, since the foam is quasi-incompressible. Since it is mostly constituted by gas, the order of magnitude of its bulk modulus is given by the atmospheric pressure, equal to $10^{5} \mathrm{~Pa}$. The order of magnitude of its shear modulus, given by $\gamma / \sqrt{A}[1]$, is much lower: with $\gamma=36 \mathrm{mN} / \mathrm{m}$ and $A=33 \mathrm{~mm}^{2}$, $\gamma / \sqrt{A} \approx 10 \mathrm{~Pa}$. The deviation of the velocity profile from (1) in the presence of wall friction is discussed in Sec. 4.2.

The fact that the velocity field of the reference experiment obeys (1) makes it a model to study the interplay between plasticity and flow. More precisely, at constant elastic stress, the deformation rate must be directly correlated to the rate of plastic events. Our experimental results strongly suggest that it is indeed true: the rate of plastic 
events, measured independently from the velocity field, depends on $r$ with a power law compatible with $1 / r^{2}$ (inset of Fig. 8), which is precisely the scaling of the deformation rate associated with the velocity field (1). Furthermore, the preferential orientations of the plastic events (Fig. 9) also support this idea: bubbles preferentially detach along the direction of elongation $\boldsymbol{e}_{r}$, whereas they preferentially attach along the direction of compression $\boldsymbol{e}_{\theta}$.

Let us go further and compare the prefactors. A single plastic event is expected to separate the two bubbles that detach by a distance of the order of the bubble size, as Fig. 2 already suggests. Hence, we may expect that the streamwise deformation rate, $\partial v_{r} / \partial r$, is of the order of the rate of plastic events times the bubble size. To test this idea, we fit the data again, but this time we fix the exponents. We fit the velocity by a law: $\log \left(-v_{r}\right)=\log c_{1}-\log r$, and the T1 frequency by a law: $\log f_{T 1}=\log c_{2}-2 \log r$. We find as best fitting parameters $c_{1}=(1.03 \pm 0.01) \times 10^{3} \mathrm{~mm}^{2} / \mathrm{s}$, and $c_{2}=25.9 \pm 2.2 \mathrm{~s}^{-1}$. The ratio of these two parameters gives a characteristic area $A_{T 1}=40 \pm 4 \mathrm{~mm}^{2}$, which is indeed very close to the bubble area $A=33.0 \mathrm{~mm}^{2}$, such that:

$$
\frac{\partial v_{r}}{\partial r}=A_{T 1} f_{T 1}
$$

This confirms quantitatively the kinematic consequence of $\mathrm{T} 1 \mathrm{~s}$, namely to incrementally shift bubbles streamwise, by inserting additional bubbles in between (Fig. 2).

It is interesting to point out that this characteristic area is a somewhat different concept from the range of plastic events which has been abundantly discussed in the context of plastic flows [35], or from the size of a plastic event which can be estimated by indirect methods like diffusive wave spectroscopy [36]. The area $A_{T 1}$ characterises the "efficiency" of a given plastic event as an increment of deformation, but does not describe its effect in its neighbourhood and the way it redistributes the elastic stress around $[37,38]$. These two kinds of information are thus complementary.

\subsection{Influence of wall friction}

\subsubsection{Velocity field}

The reference velocity field to which our results have been compared is given by (1): $\boldsymbol{v}=f(\theta) \boldsymbol{e}_{r} / r$. We have seen that while the flow of a foam constituted by SDS solution complies with such a profile, the foam made by a SLS/CAPB/LAc mixture shows significant deviations. This suggests that wall friction affects the velocity profile.

To start the discussion, we notice that (1) is a selfsimilar profile. Self-similarity is expected to hold when no characteristic length appears in the flow problem. For the flow of a Newtonian fluid in a convergent channel, the flow profile depends only on the Reynolds number $Q_{2 D} / \nu$, with $Q_{2 D}$ the flow rate per unit length, and self-similarity holds. In particular, the no-slip boundary condition does not introduce extra parameters. For the foam with the
SDS solution, the wall friction is negligible also at the side walls, hence free shear boundary condition: $\partial v_{r} / \partial \theta=0$ at $\theta= \pm \alpha_{0}$ applies to a good approximation (Fig. 6). Like the no-slip boundary condition, the free shear boundary condition is compatible with the self-similar profile (1). However, as soon as wall friction is introduced, another characteristic coefficient appears, and the self-similarity is lost.

Predicting the velocity profile for such a viscoelastoplastic material is a daunting task. However, it has been shown in pure shear with no-slip boundary condition [5] that elasticity may have a negligible effect on the velocity profile, which can then be predicted analytically from a balance between viscous stress and wall friction. On the other hand, such a simplified approach fails to predict correctly the slip velocity along smooth walls in a Poiseuille flow [6]. In our geometry, we derive the following velocity profile neglecting elasticity in Appendix A:

$v_{r}=\frac{-Q / h}{2 r \alpha_{0}-2 h \ell /(h+2 \ell)}\left(1-\frac{2 h}{h+2 \ell} \mathrm{e}^{-r \alpha_{0} / \ell} \cosh \frac{r \theta}{\ell}\right)$,

where $Q=Q_{g}+Q_{\ell}$ is the foam flow rate and $\ell$ is a characteristic length comparing the effect of wall friction and viscous stress; this expression is valid only if $\ell / r \ll 1$, which we argue is the case for SLES/CAPB/LAc mixtures in the Appendix A.

The velocity profile (3) shows that wall friction is responsible for a deviation from (1). It also captures the fact that the velocity at the side walls is lower than at the centre of the channel, because of the extra friction at the side walls. However, it predicts that $v_{r}\left(r, \theta=\alpha_{0}\right) / v_{r}(r, \theta=0)$ is a decreasing function of $r$ (see Appendix A for a proof), whereas the opposite occurs in experiments (Fig. 10) for the SLES/CAPB/LAc mixture, namely the fact that $v_{r}(r, \theta=$ $\left.\alpha_{0}\right) / v_{r}(r, \theta=0)$ increases at increasing $r$. Indeed, it is the same failure as in the Poiseuille flow of foams, namely that the slip velocity is not well predicted [6]. There can be three reasons for this discrepancy: (i) the dependencies of wall friction on velocity on one hand, and of viscous stresses on deformation rate on the other hand, may differ, (ii) the hypothesis $\ell \ll r$ breaks down before the channel exit, (iii) nonlocal effects, more precisely the redistribution of elastic stresses by plastic events, may matter.

\subsubsection{Elastic stress}

Fig. 11 shows that for the SLES/CAPB/LAc mixture, the normal elastic stress difference $\sigma_{r r}-\sigma_{\theta \theta}$ increases as the bubbles flow faster towards the exit of the channel. This effect has been previously mentioned in experiments of a foam flowing through a constriction [11]. Similarly, simulations of foam including non-quasistatic rate effects, either a variation of surface tension in the so-called vertex model [14], or an external velocity-dependent friction in the socalled viscous froth model [42], report an increase of the elastic deformation as the velocity or the deformation rate increases. 
To rationalise this fact, it has been proposed that the elastic stress tends to increase as the relaxation time $\tau_{r}$ following a plastic rearrangement becomes significant compared to the deformation rate $\dot{\varepsilon}[43]$. However, this dependence of an elastic stress on the deformation rate has been mostly discussed in simple shear, and only in theory or in simulations. We thus propose to quantify this dependence in our experiments.

First of all, we need an estimate of the deformation rate $\dot{\varepsilon}$ relevant to our experiments. The flow kinematics is more complicated than simple shear or pure elongation, but a representative deformation rate is given by the axial elongation rate, i.e. the spatial variation of the velocity along the symmetry axis. Hence, we will take $\dot{\varepsilon}=\left|\partial v_{r} / \partial r\right|_{\theta=0}$. To estimate this quantity from our measurements of velocity, we first fit $v_{r}(r, \theta=0)$ by a power law: $v_{r}(r, \theta=0)=\beta r^{\alpha}$, and we compute the deformation rate as the derivative of this fit: $\dot{\varepsilon}=\beta \alpha r^{\alpha-1}$. An advantage of our setup is that the deformation rate varies by two orders of magnitude on a given experiment between the entrance $(r=32 \mathrm{~cm})$ and the exit $(r=2.5 \mathrm{~cm})$ of the convergent channel. Since the flow is dominated by elongation, the relevant elastic quantity to study is the normal stress difference $\sigma_{r r}-\sigma_{\theta \theta}$. To make the comparison easier with other experiments and other studies, we divide the normal elastic stress difference by $\gamma / \sqrt{A}$ as in [43], and we call the resulting dimensionless quantity $\left(\sigma_{r r}-\sigma_{\theta \theta} /(\gamma / \sqrt{A})\right.$ the "elastic deformation".

Next, we quantify the relaxation time $\tau_{r}$. We proceed as described in [44], by measuring the evolution of the length of one newly created film after a T1. Even if the relaxation process might be complicated [44,45], Fig. 13 shows that the length of such a new film follows closely an exponential relaxation towards a "final" length (although this length drifts on longer times, as the bubbles concerned by the $\mathrm{T} 1$ are advected away), and the characteristic time of this exponential behaviour yields: $\tau_{r}=0.019 \mathrm{~s}$ for the SDS solution, and $0.13 \mathrm{~s}$ for the SLES/CAPB/LAc mixture. We have checked on some other examples of newly created films that these values remain correct within $30 \%$. Hence, we take these two values as good estimates of the relaxation time following a $\mathrm{T} 1$.

For the experiment with the SDS solution presented in Sec. 3.1, the deformation rate equals at most $1.7 \mathrm{~s}^{-1}$, hence $\dot{\varepsilon} \tau_{r}$ does not exceed 0.03 . Hence, the relaxation time after a $\mathrm{T} 1$ is everywhere negligible compared to the deformation rate, and the flow remains quasistatic, which is consistent with the absence of variation of the elastic stress components along the channel (Fig. 7). On the other hand, for the experiment with the SLES/CAPB/LAc mixture presented in Sec 3.2, we compute that $\dot{\varepsilon}$ increases from 0.10 to $11 \mathrm{~s}^{-1}$, hence $\dot{\varepsilon} \tau_{r}$ from 0.013 to 1.4 , along the channel. Hence, as $r$ decreases, the relaxation after plastic rearrangements is no longer short compared to the loading by the flow, which is consistent with the increase of the elastic deformation on Fig. 11.

To go further, we can test whether the elastic deformation can be rescaled by $\dot{\varepsilon} \tau_{r}$ irrespective of the location $r$ within the channel. To improve the comparison, we com-

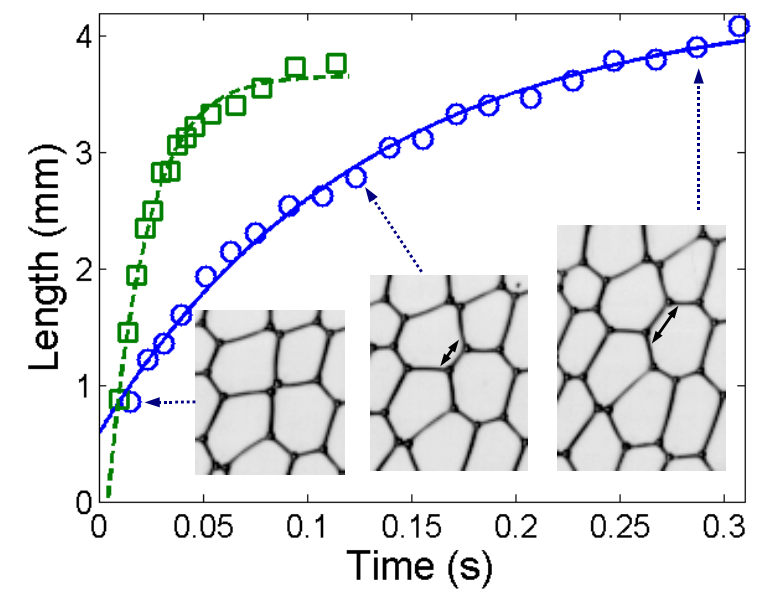

Fig. 13. Evolution of the length of a film created after a T1, for the SDS solution (squares) and the SLES/CAPB/LAc mixture (circles), and three snapshots of this film, represented by a double arrow. The time origin was chosen as the first image where this length could be measured reliably. The data were fitted by an exponential relaxation of the form $\ell(t)=\ell_{1}+$ $\ell_{2} \mathrm{e}^{-\left(t-t_{0}\right) / \tau_{r}}$, with $\ell_{1}, \ell_{2}, t_{0}$ and $\tau_{r}$ as fitting parameters; the latter parameter is identified as the relaxation time following the T1. It equals $0.019 \pm 0.001 \mathrm{~s}$ for the SDS solution, and $0.13 \pm 0.01 \mathrm{~s}$ for the SLES/CAPB/LAc mixture.

pile the results of five experiments at different gas flow rates $(100,250$ and $500 \mathrm{~mL} / \mathrm{min})$, but at the same liquid fraction $\phi_{\ell}=8.9 \%$ and the same area $A=35 \mathrm{~mm}^{2}$ within $10 \%$, and we plot the elastic deformation as a function of $\dot{\varepsilon} \tau_{r}$ in Fig. 14. This plot shows that all the data for the SLES/CAPB/LAc mixture collapse on the same master curve which is well fitted by a Herschel-Bulkleylike law: $\left(\sigma_{r r}-\sigma_{\theta \theta}\right) /(\gamma / \sqrt{A})=\sigma^{*}\left[1+\left(\dot{\varepsilon} \tau_{r} / \varepsilon^{*}\right)^{n}\right]$ with a characteristic exponent $n=0.40 \pm 0.04$. This may remind the Herschel-Bulkley law followed by the flow curves of SLES/CAPB/LAc foams [34], which exhibit a slightly lower exponent (about 0.25). However, it is irrelevant to push forward such a comparison, since we have no access to the viscous stress. The limit $\sigma^{*}$ of this law at vanishing deformation rate is also compatible with the data for the SDS solution for $\dot{\varepsilon} \tau_{r}$ lower than a few $10^{-3}$, suggesting that this regime is the quasistatic elastoplastic limit, independent on the solution except on its surface tension. However, the data for the SDS solution deviate from those with the SLES/CAPB/LAc mixture at larger $\dot{\varepsilon} \tau_{r}$, and show little dependence on $\dot{\varepsilon} \tau_{r}$. This suggests that the departure from the quasistatic elastic deformation is not universal, but depends on the viscous properties of the solution, and not only through the relaxation time after T1s.

\subsubsection{Rate of plastic events}

In Sec. 4.1, we showed that at constant elastic deformation, the rate of plastic events is proportional to the deformation rate. Since the experiment with the SLES/CAPB/LAc 


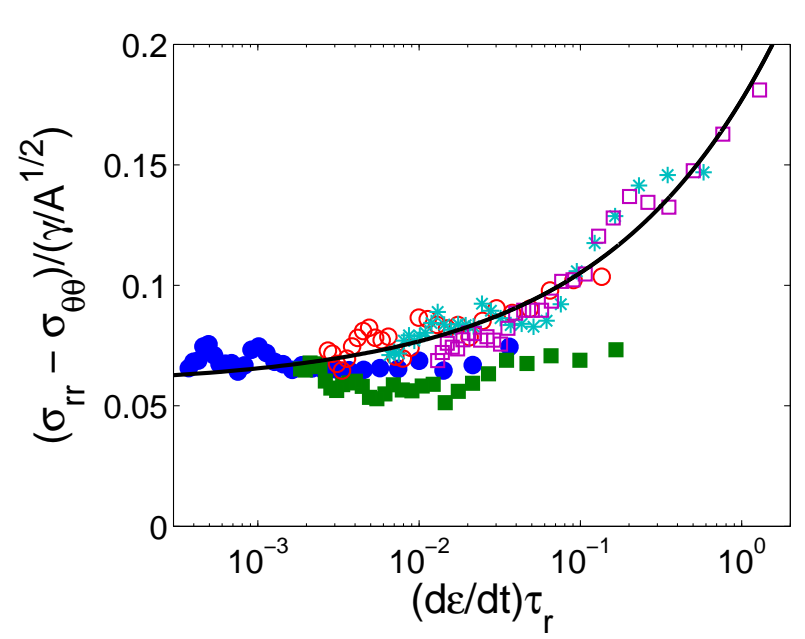

Fig. 14. Plot of the elastic deformation $\left(\sigma_{r r}-\sigma_{\theta \theta}\right) /(\gamma / \sqrt{A})$ as a function of $\dot{\varepsilon} \tau_{r}$ for foam flows with a SDS solution (full symbols) or a SLES/CAPB/LAc mixture (empty symbols), for $Q_{g}=100$ (circles), 250 (lozenges) and $500 \mathrm{~mL} / \mathrm{min}$ (squares). The curve is a single fit of all data for the SLES/CAPB/LAc mixture by a Herschel-Bulkley-like law: $\left(\sigma_{r r}-\sigma_{\theta \theta}\right) /(\gamma / \sqrt{A})=$ $\sigma^{*}\left[1+\left(\dot{\varepsilon} \tau_{r} / \varepsilon^{*}\right)^{n}\right]$, with best fitting parameters $\sigma^{*}=0.058 \pm$ $0.004, \varepsilon^{*}=0.17 \pm 0.06$ and $n=0.40 \pm 0.04$.

mixture presents a significant increase of the elastic deformation towards the channel exit (Fig. 11), it is interesting to investigate the relationship between the $\mathrm{T} 1$ frequency and the deformation rate for this experiment too.

The velocity fields depend on wall friction (Figs. 5 and 10), but overall, the deformation rate is proportional to the foam flow rate $Q$. Hence, a simple way to test whether the elastic deformation has an influence on the rate of plastic events at given deformation rate is to plot the ratio $f_{T 1} / Q$ as a function of $r$ (Fig. 15). Since $f_{T 1}$ is averaged over $\theta$, this approach is not sensitive to the angular dependence of the radial velocity discussed for the SLES/CAPB/LAc mixture (inset of Fig. 10). Fig. 15 shows that $f_{T 1} / Q$ is lower for this experiment than for the SDS solution. This clearly evidences an influence of the elastic deformation on the rate of plastic events.

Recent models $[35,46]$ claim that the rate of plastic events should be proportional, not to the sole deformation rate, but to the so-called fluidity, which is the ratio of the deformation rate to the stress. Our results are indeed in qualitative agreement with this statement. However, it is not possible to make a quantitative comparison, for the following reasons. (i) It is the total stress (elastic plus viscous) that appears in the fluidity, while we can measure only the elastic contribution. (ii) Fluidity models have been devised for pure shear only, and our flow combines shear and elongation. (iii) The extension of fluidity models in the presence of wall friction is not trivial [47, 48].

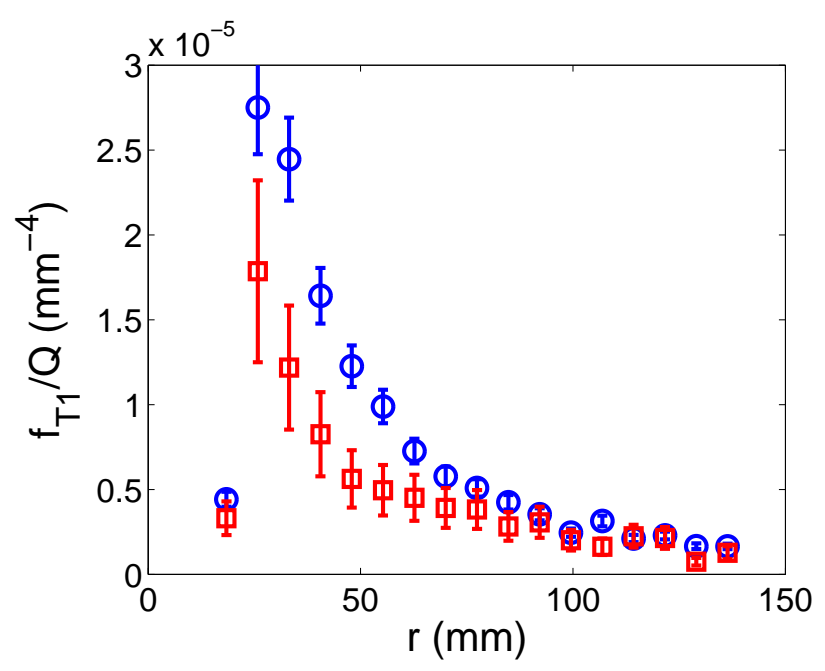

Fig. 15. Plot of the ratio of the $\mathrm{T} 1$ frequency to the foam flow rate as a function of $r$, for the experiment with the SDS solution (circles) and the SLES/CAPB/LAc mixture (squares).

\section{Conclusions}

In this paper, we have quantified in details the $2 \mathrm{D}$ flow of a foam through a convergent channel. We have showed that if wall friction is negligible, the velocity field obeys a simple self-similar form. It makes this experiment a model of plastic flow: we could establish a clear connection between the velocity field and the distribution of plastic events, showing that all of them act efficiently as increments of flow.

The use of the SLES/CAPB/LAc mixture enabled us to quantify the departure from this simple picture as wall friction becomes significant. We quantified for the first time in an experiment how the elastic deformation increases when the deformation rate increases to become of the same order as the inverse of the relaxation time following plastic events. We showed how the velocity field is modified, with a significant deceleration of the foam close to the smooth side walls, and a breakdown of the self-similar form. This qualitative picture is predicted by a model balancing wall friction and viscous stress, but this model fails to capture the variation of the ratio of the slip velocity to the centreline velocity. Finally, we showed that the elastic deformation influences the relationship between the rate of plastic events and the deformation rate.

We believe that our work opens several interesting perspectives. First, it would be interesting to find the missing ingredient to offer a full quantitative prediction of the velocity profile, including elastic effects, either in the frame of a viscoelastoplastic model [49] or as an effective nonlocal term [50]. In the same spirit but more on the experimental side, we could investigate the spatial effects of plastic events, and how they modify the elastic stress in their surroundings [38]. Moreover, if we showed that the elastic deformation was an increasing function of the deformation rate for the SLES/CAPB/LAc mixture, it was not as clear for the SDS solution: it would be interesting to understand this difference, and more precisely to iden- 
tify unambiguously the parameter that controls the departure from the quasistatic limit. Finally, we have observed in some cases that increasing even more wall friction e.g. by increasing the velocity leads to brittle fracture of the foam. Fast confined flows could therefore be an interesting alternative way to study fracture in foams.

We acknowledge discussions with Axelle Amon, Isabelle Cantat and Kirsten Martens. We thank Luca Biferale, Massimo Cencini, Alessandra Sabina Lanotte and Mauro Sbragaglia for the organisation of the workshop "Flowing Matter Across the Scales" which motivated this study.

\section{A Derivation of the velocity profile neglecting elasticity}

In the Appendix, we predict the velocity profile of a foam flowing through a convergent channel when neglecting elastic stresses. We revisit the model by Janiaud et al. [39], who first studied the interplay between viscous stress and wall friction in the context of a plane Couette flow. Because of its low density and large effective viscosity, foam has a negligible inertia in the studied flow, which is furthermore steady. With these assumptions, the model by Janiaud et al. writes:

$$
\mathbf{0}=\boldsymbol{\nabla} \cdot \tau-\nabla p+\frac{2}{h} \boldsymbol{f},
$$

where $\tau$ is the stress tensor within the foam, $p$ the pressure field and $f$ the wall friction force per unit area.

For simplicity, we assume that the viscous stresses scale linearly with the deformation rate: $\tau=\eta\left(\nabla \boldsymbol{v}+\nabla \boldsymbol{v}^{T}\right)$, with $\eta$ the foam effective viscosity, and that the wall friction force scales linearly with the velocity: $\boldsymbol{f}=-h \beta \boldsymbol{v} / 2$, with $\beta$ a friction coefficient. In reality, these scalings are sublinear [23], but nonlinear corrections were shown not to modify much flow profiles in Couette flows [40] and Poiseuille flows [6], and we expect the same here. With these assumptions, (4) becomes the Stokes equation with an additional term accounting for wall friction:

$$
\mathbf{0}=\eta \Delta \boldsymbol{v}-\nabla p-\beta \boldsymbol{v} .
$$

Because wall friction introduces a new term and a new coefficient $\beta$, self-similarity does not hold: the velocity profile (1) is not solution of (5).

As boundary conditions, we require that $\theta=0$ is an axis of symmetry, hence $\partial v_{r} / \partial \theta=0$ at $\theta=0$. Furthermore, extra friction at the side walls imposes that $\tau_{r \theta}= \pm \boldsymbol{f} \cdot \boldsymbol{e}_{r}$ at $\theta= \pm \alpha_{0}$, hence:

$$
\frac{1}{2} h \beta v_{r}=\mp \frac{\eta}{r} \frac{\partial v_{r}}{\partial \theta}, \quad \theta= \pm \alpha_{0} .
$$

Finally, conservation of flow rate imposes the integral condition:

$$
Q=-h \int_{-\alpha_{0}}^{\alpha_{0}} r v_{r} \mathrm{~d} \theta,
$$

where $Q$ is the volumetric flow rate, as defined in the experiments. The minus sign reminds that when $Q>0$, then $v_{r}<0$.
To solve the problem, we take the curl of (5) to remove the pressure. For a $2 \mathrm{D}$ flow, the vorticity $\boldsymbol{\omega}=\boldsymbol{\nabla} \times \boldsymbol{v}$ is a scalar field: $\boldsymbol{\omega}=\omega \boldsymbol{e}_{z}$, hence we get:

$$
\eta \Delta \omega-\beta \omega=0 .
$$

This is a Helmholtz equation which can be solved analytically [41], but we will content ourselves with a qualitative discussion and an approximate solution.

First, if wall friction is negligible as is the case for SDS solution, then Eq. (8) reduces to $\Delta \omega=0$, and the boundary condition (6) reduces to $\partial v_{r} / \partial \theta=0$ at $\theta=$ $\pm \alpha_{0}$. Together with the symmetry condition $\partial v_{r} / \partial \theta=0$ at $\theta=0$, this imposes that $v_{r}$ does not depend on $\theta$. Therefore, the conservation of flow rate (7) imposes the velocity profile:

$$
v_{r}=-\frac{Q}{2 \alpha_{0} h r}
$$

which is a particular case of the self-similar law (1).

If wall friction is not negligible, Eq. (8) contains a characteristic length: $\ell=\sqrt{\eta / \beta}$ which is the shear localisation length for the pure shear flow of confined foams [39,5]. In the convergent geometry, it is relevant to compare it with the distance $r$. In our experiments, we have seen that the exit of the channel is at $r=2.5 \mathrm{~cm}$. In previous experiments with comparable foams [6], the length $\ell$ was found to be around $0.5 \mathrm{~mm}$. Hence, we now focus on the case $\ell / r \ll 1$.

A naive inspection of (8) suggests the ratio of the viscous term $\eta \Delta \omega \approx \eta \omega / r^{2}$ to the wall friction term is of order $\ell^{2} / r^{2} \ll 1$, hence wall friction dominates and (8) reduces simply to:

$$
\omega=0,
$$

i.e. an irrotational flow. However, this is incompatible with the boundary condition (6), which shows that sidewall friction is a source of vorticity. Therefore, there is a shear boundary layer of thickness $\ell$ close to the side walls where friction and viscous stresses balance.

In such boundary layers, since $\ell \ll r$, the axial variation of the vorticity is negligible compared to its angular variation, and (8) simplifies into:

$$
\frac{1}{r^{2}} \frac{\partial^{2} \omega}{\partial \theta^{2}}-\frac{\omega}{\ell^{2}}=0
$$

where $r$ can be treated as a constant. Hence, the vorticity has an exponential profile: $\omega=\mp \omega_{0} \mathrm{e}^{-r\left(\alpha_{0} \mp \theta\right) / \ell}$ close to the side wall $\theta= \pm \alpha_{0}$, which matches the irrotational flow (9) as $r\left(\alpha_{0} \mp \theta\right) / \ell \gg 1$. Hence, the vorticity profile equals:

$\omega=\omega_{0}\left[-\mathrm{e}^{-r\left(\alpha_{0}-\theta\right) / \ell}+\mathrm{e}^{-r\left(\alpha_{0}+\theta\right) / \ell}\right]=-2 \omega_{0} \mathrm{e}^{-r \alpha_{0} / \ell} \sinh \frac{r \theta}{\ell}$.

Similar to the Laplacian term, the general relation between the vorticity and the velocity:

$$
\omega=\frac{1}{r}\left[\frac{\partial}{\partial r}\left(r v_{\theta}\right)-\frac{\partial v_{r}}{\partial \theta}\right]
$$


simplifies to $\omega=-\frac{1}{r} \frac{\partial v_{r}}{\partial \theta}$, hence $v_{r}=v_{0}+2 \ell \omega_{0} \mathrm{e}^{-r \alpha_{0} / \ell} \times$ $\cosh (r \theta / \ell)$. Inserting this expression in the boundary condition (6) yields a first relation between the two unknowns $\omega_{0}$ and $v_{0}: \omega_{0}=-h v_{0} /\left(2 \ell^{2}+\ell h\right)$. The second relation comes from the conservation of flow rate ( 7$)$. Finally, the velocity field is:

$$
v_{r}=\frac{-Q / h}{2 r \alpha_{0}-2 h \ell /(h+2 \ell)}\left(1-\frac{2 h}{h+2 \ell} \mathrm{e}^{-r \alpha_{0} / \ell} \cosh \frac{r \theta}{\ell}\right),
$$

which strictly speaking is valid only if $\ell / r \ll 1$, and up to corrections of order $\ell / r$.

Using this equation, it is straightforward to show that:

$$
\frac{v_{r}\left(r, \theta=\alpha_{0}\right)}{v_{r}(r, \theta=0)}=\frac{h+2 \ell-h\left(1+\mathrm{e}^{-2 r \alpha_{0} / \ell}\right)}{h+2 \ell-2 h \mathrm{e}^{-r \alpha_{0} / \ell}},
$$

and that:

$$
\begin{aligned}
\frac{\mathrm{d}}{\mathrm{d} r}\left[\frac{v_{r}\left(r, \theta=\alpha_{0}\right)}{v_{r}(r, \theta=0)}\right]= & -\frac{2 \alpha_{0} h}{\ell} \frac{\mathrm{e}^{r \alpha_{0} / \ell}}{\left[(h+2 \ell) \mathrm{e}^{r \alpha_{0} / \ell}-2 h\right]^{2}} \\
& \times\left(1-\mathrm{e}^{-r \alpha_{0} / \ell}\right)\left(2 \ell-h \mathrm{e}^{-r \alpha_{0} / \ell}\right),
\end{aligned}
$$

which is negative for all values of $r>0$ if $2 \ell>h$. If $2 \ell<h$, this derivative is negative if $\mathrm{e}^{-r \alpha_{0} / \ell}<2 \ell / h$, i.e. $r>-\frac{\ell}{\alpha_{0}} \ln \frac{2 \ell}{h}$. The latter quantity does not exceed $0.368 h / 2 \alpha_{0}=0.8 \mathrm{~mm}$ (the factor 0.368 is the opposite of the minimum value taken by the function $x \ln x$ for $x$ between 0 and 1). Hence, over our experimental range of $r$, the velocity profile (3) implies that $v_{r}\left(r, \theta=\alpha_{0}\right) / v_{r}(r, \theta=$ 0 ) is a decreasing function of $r$.

\section{References}

1. I. Cantat, S. Cohen-Addad, F. Elias, F. Graner, R. Höhler O. Pitois, F. Rouyer, A. Saint-Jalmes, edited by S. J. Cox, Foams, Structure and Dynamics (Oxford University Press, 2013).

2. G. K. Batchelor, J. Fluid Mech. 41, (1970) 545-570.

3. C. Raufaste, B. Dollet, K. Mader, S. Santucci, R. Mokso, EPL 111, (2015) 38004.

4. Y. Wang, K. Krishan, M. Dennin, Phys. Rev. E 73, (2006) 031401.

5. G. Katgert, M. E. Möbius, M. van Hecke, Phys. Rev. Lett. 101, (2008) 058301.

6. B. Dollet, A. Scagliarini, M. Sbragaglia, J. Fluid Mech. 766, (2015) 556-589.

7. B. Dollet, M. Aubouy, F. Graner, Phys. Rev. Lett. 95 (2005) 168303.

8. B. Dollet, M. Durth, F. Graner, Phys. Rev. E 73, (2006) 061404 .

9. B. Dollet, F. Graner, J. Fluid Mech. 585, (2007) 181-211.

10. Y. Bertho, C. Becco, N. Vandewalle, Phys. Rev. E 73, (2006) 056309.

11. B. Dollet, J. Rheol. 54, (2010) 741-760.

12. T. Okuzono, K. Kawasaki, Phys. Rev. E 51, (1995) 12461253.

13. D. J. Durian, Phys. Rev. Lett. 75, (1995) 4780-4783.

14. I. Cantat, Soft Matter 7, (2011) 448-455.
15. C. Raufaste, B. Dollet, S. Cox, Y. Jiang, F. Graner, Eur. Phys. J. E 23, (2007) 217-228.

16. I. T. Davies, S. J. Cox, Colloids Surf. A 344, (2009) 8-14. 17. I. Cheddadi, P. Saramito, B. Dollet, C. Raufaste, F. Graner, Eur. Phys. J. E 34, (2011) 1.

18. F. Boulogne, S. J. Cox, Phys. Rev. E 83, (2011) 041404.

19. S. A. Jones, B. Dollet, N. Slosse, Y. Jiang, S. J. Cox, F. Graner, Colloids Surf. A 382, (2011) 18-23.

20. V. J. Langlois, J. Rheol. 58, (2014) 799-818.

21. D. A. Reinelt, A. M. Kraynik, J. Fluid Mech. 311, (1996) 327-343.

22. D. A. Reinelt, A. M. Kraynik, J. Rheol. 44, (2000) 453471.

23. N. D. Denkov, S. Tcholakova, K. Golemanov, K. P. Ananthapadmanabhan, A. Lips, Soft Matter 5, (2009) 3389-3408.

24. S. Arif, J. C. Tsai, S. Hilgenfeldt, EPL 92, (2010) 38001.

25. S. Arif, J. C. Tsai, S. Hilgenfeldt, J. Rheol. 56, (2012) 485-499.

26. I. Ben Salem, I. Cantat, B. Dollet, J. Fluid Mech. 714, (2013) 258-282.

27. J. C. Earnshaw, A. H. Jaafar, Phys. Rev. E 49, (1994) 5408-5411.

28. D. Chen, K. W. Desmond, E. R. Weeks, Soft Matter 8, (2012) 10486-10492.

29. D. Chen, K. W. Desmond, E. R. Weeks, Phys. Rev. E 91, (2015) 062306.

30. G. B. Jeffery, Phil. Mag. 29, (1915) 455-465.

31. G. Hamel, Jahresber. Deutsch. Math.-Verein. 25, (1916) 34-60.

32. K. Pohlhausen, Z. Angew. Math. Mech. 1, (1921) 252-268.

33. L. Landau, E. Lifshitz, Mécanique des fluides, 3ème édition (Mir, 1994).

34. K. Golemanov, N. D. Denkov, S. Tcholakova, M. Vethamuthu, A. Lips, Langmuir 24, (2008) 9956-9961.

35. L. Bocquet, A. Colin, A. Ajdari, Phys. Rev. Lett. 103, (2009) 036001.

36. D. J. Durian, D. A. Weitz, D. J. Pine, Science 252, (1991) 686-.

37. S. J. Cox, F. Graner, M. F. Vaz, Soft Matter 4, (2008) 1871-1878.

38. K. W. Desmond, E. R. Weeks, Phys. Rev. Lett. 115, (2015) 098302.

39. E. Janiaud, D. Weaire, S. Hutzler, Phys. Rev. Lett. 97, (2006) 038302.

40. D. Weaire, S. Hutzler, V. J. Langlois, R. J. Clancy, Phil. Mag. Lett. 88, (2008) 387-396.

41. A. Prosperetti, Advanced Mathematics for Applications (Cambridge University Press, 2011).

42. B. Embley, P. Grassia, Colloids Surf. A 382, (2011) 8-17.

43. P. Marmottant, F. Graner, Soft Matter 9, (2013) 96029607.

44. M. Durand, H. A. Stone, Phys. Rev. Lett. 97, (2006) 226101.

45. A. L. Biance, S. Cohen-Addad, R. Höhler, Soft Matter 5, (2009) 4672-4679.

46. V. Mansard, A. Colin, P. Chaudhuri, L. Bocquet, Soft Matter 9, (2013) 7489-7500.

47. J. D. Barry, D. Weaire, S. Hutzler, Phil. Mag. Lett. 91, (2011) 432-440.

48. A. Scagliarini, B. Dollet, M. Sbragaglia, Colloids Surf. A 473, (2015) 133-140.

49. P. Saramito, J. Non-Newtonian Fluid Mech. 145, (2007) $1-14$.

50. J. Goyon, A. Colin, G. Ovarlez, A. Ajdari, L. Bocquet, Nature 454, (2008) 84-87. 